Aim/Background: BPDCN is a rare aggressive haematological malignancy in the elderly, with high frequency of cutaneous and bone marrow involvement and a poor prognosis. Our aims are (1) to increase awareness of this rare entity and (2) discuss the value of different investigation tools in diagnosis and response assessment. Method: A 75-year-old man presented with widespread nodular violaceous rash. Full blood count showed Hb 112 g/L, WCC $16.5 \times 10^{9} / \mathrm{L}$, platelets $162 \times 10^{9} / \mathrm{L}$ and circulating blasts. Skin biopsy, bone marrow biopsy and flow cytometry were consistent with BPDCN (CD4+, CD56+, CD38+, CD45dim, CD123bright, negative for lineage markers). He was commenced on induction chemotherapy using a modified acute lymphoblastic leukaemia (ALL) protocol for the elderly.

Results: Chemotherapy was well tolerated with complete resolution of skin lesions by day 15 . Repeat bone marrow biopsy (day 30) revealed significant reduction but persistence of blasts (9\%) detected by flow cytometry.

Conclusions: Ancilliary investigations in particular flow cytometry and immunohistochemistry are essential in the diagnosis of BPDCN and to distinguish this from other haematological malignancies. Bone marrow biopsy together with flow cytometry and/or immunohistochemistry to assess remission status should be considered post induction chemotherapy even if cutaneous manifestations have resolved.

\section{WILMS TUMOUR GENE 1 (WT1) MUTATIONS IN ACUTE MYELOID LEUKEMIA (AML)}

Joëlle Marivel $^{1,2}$, Anabel Kearney ${ }^{1,2}$, Anna Nelson $^{1}$, Silvia Ling ${ }^{1,2}$

${ }^{1}$ Liverpool Hospital, Liverpool, and ${ }^{2}$ Ingham Institute for Applied Medical Research, Liverpool, NSW, Australia

Background: The role of WT1 mutations in the pathogenesis of AML is unclear but there is evidence to suggest they are associated with a poor prognosis and response to treatment. We show that screening for WT1 mutations is feasible in a diagnostic laboratory and aim to correlate the WT1 mutations with clinical characteristics, morphology, karyotype, other genetic mutations, response to treatment and survival.

Method: Mutations in exons 7 and 9 of the WT1 gene were detected by fragment analysis. To confirm the results, 20 samples were re-tested by Sanger sequencing and next generation sequencing.

Results: Three out of 50 AML patients were positive for mutations in exon 7. All 3 patients had normal karyotype and treated with induction chemotherapy $7+3$ and HiDAC consolidation. Patient 1 survived 12 months and died of relapsed AML. Patient 2 had an allogeneic stem cell transplant at 2 nd CR but died from severe GVHD 14 months from diagnosis. Patient 3 is still alive at 12 months having had an allogeneic stem cell transplant in 1st CR. Conclusion: WT1 mutation detection is feasible in the routine diagnostic setting. In our cohort they are associated with a poor prognosis, but further studies are still required.

\section{TRISOMY 3 AND 18 ASSOCIATED WITH WALDENSTROM MACROGLOBULINAEMIA: A SMALL CASE SERIES OF THREE PATIENTS}

Gregory Orchard, Helen Wordsworth, Ross Brookwell Sullivan Nicolaides Pathology, Taringa, Qld, Australia
Waldenstrom macroglobulinaemia (WM), an IgM-secreting lymphoplasmacytic lymphoma (LPL), is a neoplasm of small B lymphocytes, plasmacytoid lymphocytes, and plasma cells involving bone marrow and sometimes lymph nodes and spleen. The predominant cytogenetic abnormality reported in WM is deletion $6 \mathrm{q}$ with one study reporting a frequency of up to $63 \%$. There are infrequent reports of trisomy 3 , trisomy 18 , or the combined trisomy 3 and 18 .

In this small case series, we describe three cases of WM presenting from 2010-2015. All three cases had bone marrow morphological features of LPL and an IgM paraprotein and all cases were found to have both trisomy 3 and trisomy 18 on conventional karyotyping.

\section{THE INCIDENCE, CLINICAL AND PROGNOSTIC SIGNIFICANCE OF PLASMA CELL DYSCRASIAS WITH ABERRANT CD4 AND/OR CD8+ EXPRESSION}

Gregory Orchard $^{1}$, Johan Niemann ${ }^{2}$, Cyriac Abraham ${ }^{2}$, Raymond Banh ${ }^{1}$

${ }^{1}$ Mater Health Service, and ${ }^{2}$ Mater Pathology, Brisbane, Qld, Australia

Aim: To determine: (a) the proportion of patients diagnosed with plasma cell dyscrasias at our institution who had CD4 and/or CD8 expressing plasma cells by flow cytometry, and (b) any association between this aberrant phenotype and clinical and prognostic factors.

Method: We interrogated our pathology database to identify patients with a plasma cell dyscrasia diagnosed between 2008-2014, with plasma cells detected by flow cytometry of bone marrow. These patients' records were reviewed to determine if their plasma cells expressed CD4 and/or CD8, which was assessed against relevant prognostic and clinical factors.

Results: 74 specimens were evaluable, of which 17 (23\%) were CD4 positive. No cases were CD8 positive. The age and sex of CD4 positive and negative patients were comparable. There was a significant association of CD4 positivity with plasma cell myeloma, particularly relapsed disease; ISS stage II or III; higher LDH level; and high risk by combined karyotyping and FISH analysis. There was also a trend towards higher marrow plasma cell burden.

Conclusion: CD4 positivity is associated with higher risk disease and factors associated with a poor prognosis. It is unclear whether CD4 status is a useful prognostic or predictive factor or represents clonal evolution of plasma cells.

\section{FULL BLOOD COUNT - INTERNAL QC PROTOCOL, A REVIEW BY THE RCPAQAP HAEMATOLOGY}

John Sioufi, Tony Badrick, Leanne Sinclair

RCPAQAP Haematology, St Leonards, NSW, Australia

Aim: The RCPAQAP Haematology has undertaken an exercise to review the internal quality control protocol for FBC instrumentation as well as review the action taken by laboratories when non-conforming results are evident in the RCPAQAP proficiency testing reports.

Method: A questionnaire was sent to laboratories enrolled in the RCPAQAP FBC module. Laboratories were asked to provide 